\title{
Nordiques
}

35 | 2018

Tove Jansson : Par delà les genres. Pratiques

linguistiques dans le Norden du XXIe siècle : Quels

enjeux sociaux?

\section{Tove Jansson et la philosophie de l'eau}

\section{Kuisma Korhonen}

\section{(2) OpenEdition \\ Journals}

\section{Édition électronique}

URL : https://journals.openedition.org/nordiques/810

ISSN : 2777-8479

Éditeur :

Association Norden, Bibliothèque de Caen la mer

\section{Édition imprimée}

Date de publication : 1 mai 2018

Pagination : 43-53

ISBN : 979-1-0959140-1-3

ISSN : $1761-7677$

\section{Référence électronique}

Kuisma Korhonen, «Tove Jansson et la philosophie de l'eau », Nordiques [En ligne], 35 | 2018, mis en ligne le 02 février 2021, consulté le 06 octobre 2021. URL : http://journals.openedition.org/nordiques/ 810 


\section{Tove Jansson \\ et la philosophie de l'eau}

Kuisma Korhonen*

\section{RÉSUMÉ}

La mer et l'élément aquatique jouent souvent un rôle significatif dans l'auvre de Tove Jansson, permettant à l'auteur une réflexion sur les grands thèmes de la philosophie occidentale. Cet article retrace les intertextes philosophiques de Jansson et étudie sa philosophie de l'eau comme philosophie de la vie. Quatre aspects du thème sont soumis à l'analyse : l'eau en tant qu'élément de changement perpétuel; la mer en tant qu'invitation à l'aventure et à la découverte ; l'océan en tant qu'élément séduisant, mais aussi dangereux; et l'eau et la mer comme source génératrice, éléments créatif et féminin de l'imagination.

\section{ABSTRACT}

In Tove Jansson's works, the sea and water often play a meaningful role, giving the writer the opportunity to reflect on the great themes of western philosophy. This article follows Jansson's philosophical subtexts and studies her philosophy of water as a philosophy of life. Four aspect of the topic are analysed: water as a constantly changing element; the sea as an invitation to adventure and exploration; the ocean as a seductive yet dangerous element; and water and the sea as sources, creative and feminine elements of imagination.

* Kuisma Korhonen est professeur de littérature à l'université d'Oulu, Finlande. 
Le premier livre publié par Tove Jansson, en 1933, sous le pseudonyme de Vera Haij, s'intitule Sara och Pelle och Neckens bläckfiskar (Sara et Pelle et les pieuvres du Näcken) - une petite aventure sous-marine illustrée qui met en scène deux enfants jouant avec des pieuvres et créatures fantastiques ${ }^{1}$. Son dernier ouvrage, Anteckningar från en ö (Notes d'une île), paru en 1996 et illustré par Tuulikki Pietilä, avait aussi le milieu marin pour cadre : l'île de Klovharu dans le golfe de Finlande, où Tove Jansson et Tuulikki Pietilä ont passé leurs étés ${ }^{2}$. Dans l'œuvre et la carrière extraordinaire de Tove Jansson située entre ces deux titres, la mer et l'élément aquatique jouent souvent un rôle important, aussi bien dans les aventures des Moumine que dans ses livres soi-disant pour adultes. Tove Jansson a aimé la mer toute sa vie.

L'eau, la mer et l'océan sont aussi des symboles culturels très chargés dans la philosophie occidentale 3 . Tove Jansson a passé son enfance dans un environnement artistique et intellectuel ; sa famille et ses amis discutaient souvent de questions philosophiques. La première image connue de la figure du troll Snork, qui allait plus tard devenir Moumine, était un croquis fait pour illustrer une discussion (écrit sur un mur de toilettes) entre Tove et son frère sur la philosophie d'Emmanuel Kant ${ }^{4}$.

Dans cet article, je vais étudier les dimensions philosophiques dans l'œuvre de Jansson et interpréter sa philosophie de l'eau comme philosophie de la vie. Je veux m'attacher en particulier à trois dimensions : l'eau en tant qu'élément de changement perpétuel ; la mer en tant qu'invitation à l'aventure et à la découverte ; et l'océan en tant qu'élément séduisant, mais aussi dangereux. Ces trois dimensions correspondent, en fait, au rôle de l'eau dans les philosophies d'Héraclite, de Hegel et de Nietzsche. Cependant, nous pouvons également ajouter une quatrième dimension à ces analyses, qu'on trouve plus dans le folklore et la poésie que dans la philosophie canonique : l'eau et la mer en tant que source génératrice, éléments créatif et féminin de l'imagination.

\section{TROIS DIMENSIONS PHILOSOPHIQUES DE L'ÉLÉMENT AQUATIQUE : Héraclite, Hegel, Nietzsche}

Pour Héraclite, l'eau était un élément de transformation perpétuelle du monde. La continuité et le changement représentent les deux côtés du même processus de devenir :

On ne peut pas entrer une seconde fois dans le même fleuve, car c'est une autre eau qui vient à vous; elle se dissipe et s'amasse de nouveau ; elle recherche

1 Tove Jansson [pseud. Vera Haij], Sara och Pelle och Neckens bläckfiskar, Helsingfors, Bildkonst, 1933.

2 Tove Jansson, Anteckningar från en $\ddot{o}$, Stockholm, Bonniers, 1996.

3 Pour l'histoire culturelle de l'eau, voir Kulturgeschichte des Wassers, H. Böhme (éd.), Francfort-sur-leMain, Suhrkamp, 1988.

4 Boel Westin, Tove Jansson. Ord, bild, liv, Helsingfors, Schildts, 2007, p. 167. 
et abandonne, elle s'approche et s'éloigne. Nous descendons et nous ne descendons pas dans ce fleuve, nous y sommes et nous n'y sommes pas.

L'ontologie héraclitienne du monde comme transformation perpétuelle a souvent, dans l'histoire de la pensée occidentale, conduit à un certain scepticisme épistémologique. Rien de certain ne peut être dit du monde, puisque le sujet et l'objet sont en mouvement incessant. On va voir que c'est aussi le cas chez Jansson.

Dans la vision héraclitienne, aucun progrès réel n'est possible : le changement continu est également répétitif et circulaire. La vision de Hegel, au contraire, se fonde sur la possibilité du progrès radical. Dans la dialectique du devenir historique, la mer représente le royaume de la liberté, mais aussi celui de la découverte et du commerce. Pour Hegel, la mer était une vision d'infini, quelque chose que l'homme occidental doit conquérir :

La mer, donne d'une façon générale naissance à un type de vie spécial. L'élément indéterminé nous donne l'idée de l'illimité et de l'infini, et l'homme, en se sentant au milieu de cet infini, en tire courage pour dépasser le limité. La mer elle-même est ce qui n'a pas de bornes et ne tolère pas, comme la terre ferme, les pacifiques délimitations en cités. La terre, la plaine fluviale, fixe l'homme au sol. Sa liberté est ainsi restreinte par un immense ensemble de liens. Mais la mer le conduit au-delà de cette limitation. La mer éveille le courage, elle invite l'homme à la conquête, au brigandage, mais aussi au gain et à l'acquisition. ${ }^{6}$

Mais la mer est aussi un élément dangereux. La liberté qu'un océan semble nous promettre peut également signifier la perte de vieilles vérités. L'aventure maritime marque en outre la perte de la terre, comme Friedrich Nietzsche nous le rappelle :

Dans l'horizon de l'infini. - Nous avons quitté la terre et sommes montés à bord ! Nous avons brisé le pont qui était derrière nous, - mieux encore, nous avons brisé la terre qui était derrière nous ! Eh bien ! petit navire, prends garde ! À tes côtés, il y a l'océan : il est vrai qu'il ne mugit pas toujours, et parfois sa nappe s'étend comme de la soie et de l'or, une rêverie de bonté. Mais il viendra des heures où tu reconnaîtras qu'il est infini et qu'il n'y a rien de plus terrible que l'infini. Hélas! pauvre oiseau, toi qui t'es senti libre, tu te heurtes maintenant aux barreaux de cette cage ! Malheur à toi, si tu es saisi du mal du pays de la terre, comme s'il y avait eu là plus de liberté, - et maintenant il n'y a plus de « terre " !

5 Alfred Fouillée, Extraits des grands philosophes, Paris, Librairie Delagrave, 1938, p. 25.

6 Georg W. F. Hegel, La raison dans l'histoire, trad. de K. Papaioannou, Paris, Plon, 1965, p. 228.

7 Friedrich Nietzsche, Le gai savoir, trad. de H. Albert, Paris, Bibliothèque nationale de France (Collection XIX), 2016, $\$ 124$. 
Comme Tuula Karjalainen le montre dans la biographie qu'elle a consacrée à l'auteur, Tove Jansson connaissait bien la philosophie de Nietzsche ${ }^{8}$. Son ami et amant Atos Virtanen, philosophe et homme politique de gauche, a publié en 1945 l'étude Nietzsche den otidsenlige (Nietzsche l'anachronique). Comme Tove l'avoue dans ses lettres, elle a trouvé l'enthousiasme d'Atos pour le philosophe allemand parfois amusant, parfois quelque peu lassant, bien qu'elle ait aimé sa vitalité, sa liberté et sa joie de vivre. De plus, c'est Atos qui a encouragé Tove à publier son premier livre sur Moumine, et il semble clair que la figure de Snusmumrik (ou le Renaclerican, comme il est nommé en français) est inspirée d'Atos qui était un esprit libre - trop libre, en fait, pour nouer des liens permanents avec d'autres.

Bien que Tove (comme Atos lui-même) critiquât certains aspects de la pensée de Nietzsche, on peut voir qu'elle partageait quelque chose du ton libre et affirmatif de l'auteur du Gai savoir. De plus, c'est ce même ton que l'on peut trouver chez un penseur que Nietzsche appréciait beaucoup, Michel de Montaigne. Comme lui, Nietzsche se voyait tel quelqu'un qui avait traversé le scepticisme philosophique et trouvait, à la fin, un ton affirmatif à la vie. Et c'était aussi la philosophie de Tove Jansson : vivre au sein d'un monde en mouvement, dans l'incertitude profonde, avec un esprit libre et affirmatif.

Pour lui, la mer n'était pas uniquement une surface, mais aussi des profondeurs mystérieuses. D'une certaine manière, Jansson était comme "l'amant marine " de Luce Irigaray, une voix féminine qui rappelait au chercheur de vérité masculin (Hegel ou Nietzsche) que la mer existait non seulement comme une chose à conquérir, mais encore qu'elle avait sa propre âme et sa propre logique. De plus, on peut voir dans la mer de Jansson un élément de créativité et d'imagination bachelardienne, ainsi que la mobilité héraclitienne de l'eau qui se donne aux rêveries profondes et surprenantes ${ }^{10}$.

\section{LE MONDE FLOTTANT}

Dans le premier Moumine, Småtrollen och den stora översvämningen (Moumine et la grande inondation), l'eau est surtout un élément de menace et de destruction ${ }^{11}$. Dans ce petit récit que Jansson ne voulut pas inclure par la suite dans le canon des Moumine, la famille fuit un grand déluge. Plus tard, l'auteur revient au motif du déluge dans Farlig Midsommar (L’été dramatique de Moumine) ${ }^{12}$. L'eau y transforme le monde habituel. La cuisine de la maison des Moumines est engloutie et

8 Tuula Karjalainen, Tove Jansson. Tee työtä ja rakasta, Helsinki, Tammi, 2013, p. 89-94.

9 Atos Virtanen, Nietzsche den otidsenlige, Stockholm, Bonniers, 1945.

10 Gaston Bachelard, L'eau et les rêves. Essai sur l'imagination de la matière, Paris, José Corti, 1942.

11 Tove Jansson, Småtrollen och den stora översvämningen, Helsingfors, Schildts, 1945.

12 Tove Jansson, Farlig Midsommar, Helsingfors, Schildts, 1954. 
Moumine le Troll doit plonger pour trouver les ustensiles de leur petit-déjeuner. La famille quitte alors la maison et trouve un bâtiment étrange, un théâtre flottant.

On peut voir, dans la vision de ce théâtre flottant, une figure métafictive. Souvent, les Moumine sont classés en deux catégories : les premiers seraient épisodiques et concentrés sur les aventures, les seconds des romans plus psychologiques. Je propose cependant de diviser l'épopée Moumine en trois catégories : entre les purs récits d'aventure, d'une part et les récits plus ou moins psychologiques comme Trollvinter (Un hiver dans la vallée de Moumine) ${ }^{13}$ ou Pappa och havet (Papa Moumine et la mer) ${ }^{14}$ d'autre part, on peut situer des livres plus ou moins métafictifs. Dans Mominpappa's bravader (surtout dans sa version finale Mominpappa's memoarer, Les mémoires de papa Moumine) ${ }^{15}$, Farlig Midsommar, tout comme dans le livre illustré Hur gick det sen? (Que crois-tu qu'il arriva?) ${ }^{16}$, c'est surtout l'acte et les conditions de narration et de représentation qui sont thématisés. Tove Jansson se tourne vers son propre métier de productrice de livres et d'images, de créatrice de représentations, et nous montre l'acte de raconter (Mominpappa's memoarer), le spectacle dramatique (Farlig Midsommar), et le livre comme un objet physique (Hur gick det sen ?).

Le théâtre flottant n'était pas une invention de Tove Jansson. On connaît un théâtre flottant vénitien, Teatro deto il mondo, projeté par Vincenzo Scamozzi en 1593 pour le couronnement de la femme du doge Morosina Grimani (on peut en trouver une illustration au Museo Correr à Venise). Le nom de ce théâtre fait référence à l'ancien topos du theatrum mundi - le monde entier vu comme un théâtre, une figure utilisée quelques années plus tard par William Shakespeare et ses collègues pour nommer leur théâtre Globe. Le monde est, selon ce topos, un immense jeu d'illusions où chaque personne joue son rôle.

Le fait que le théâtre soit flottant, que le jeu des illusions soit fondé sur un socle instable et mouvant, peut être vu aussi comme une référence à la tradition médiévale de la nef des fous. D'une certaine façon, on peut voir le monde entier comme tel. On peut aussi y voir une vision du monde plus ou moins héraclitienne ou sceptique, partagée par plusieurs penseurs à la fin du $\mathrm{XVI}^{e}$ siècle, comme par Michel de Montaigne dans ses Essais: «Le monde n'est qu'une branloire pérenne : toutes choses y branlent sans cesse, la terre, les rochers du Caucase, les pyramides d'Égypte, et du branle public, et du leur. La constance même n'est autre chose

13 Tove Jansson, Trollvinter, Helsingfors, Schildts, 1957.

14 Tove Jansson, Pappa och havet, Helsingfors, Schildts, 1965.

15 Tove Jansson, Mominpappa's bravader. Skrivna av honom själv, Helsinki, Schildts, 1956 ; Tove Jansson, Mominpappa's memoarer, Helsingfors, Schildts, 1968.

16 Tove Jansson, Hur gick det sen? Boken om Mymlan, Mumintrollet och Lilla My, Helsingfors, Schildts, 1952. 
qu'un branle plus languissant. $»^{17}$ Le monde entier était, pour Montaigne, tel un océan, toujours en mouvement, mystérieux et inconnaissable.

Le golfe de Finlande est une petite extension de la mer Baltique et non pas un océan, mais dans l'échelle des Moumines, c'est bien un océan - on ne sait rien de ce qui existe de l'autre côté. Et même dans les romans et les nouvelles pour adultes de Jansson, le golfe de Finlande se présente presque comme un océan, tumultueux et sans limites précises.

Non seulement la littérature de la Renaissance, mais aussi la littérature postmoderne de notre temps nous montrent une vision antifondationnaliste du monde. On a relevé des influences modernistes chez Jansson, mais on peut aussi établir un lien, un peu surprenant peut-être, entre la figure du théâtre flottant de Farlig Midsommar et celle de l'opéra flottant de John Barth dans son roman postmoderne devenu classique, Floating Opera, publié un an plus tard, en 1955. Le narrateur Todd Andrews y voit le monde comme un opéra flottant et les hommes peuvent apercevoir seulement des bribes du spectacle de la vie parce que l'opéra continue d'avancer avec le flux de l'eau et du temps. (Cette image a été inspirée par de vrais théâtres flottants, des showboats, qui ont l'habitude de voyager le long de la rivière Mississippi, par exemple le Floating Theatre de James Adams.) Les deux livres sont, bien évidemment, très différents quand on pense à leur atmosphère - le jeu agréable de Jansson contre le nihilisme de Barth -, mais on peut, dans ces deux œuvres, interpréter la figure du théâtre flottant comme une figure métatextuelle. Le monde est un jeu d'illusions sans autre fondation que l'eau, l'élément du mouvement et de la transformation constante. Pour les héros de John Barth, cette situation est une source d'anxiété et d'indécision; pour les héros de Tove Jansson, comme pour Montaigne, c'est une source de liberté.

\section{LE MONDE EN MOUVEMENT PERPÉTUEL}

La fragilité du monde se présente, en des termes plus sinistres, dans le premier récit officiel de l'épopée des Moumines, Kometjakten (Une comète au pays de Moumine $)^{18}$. Dans ce récit, l'approche de la comète fait disparaitre la mer, et Moumine et ses amis peuvent marcher sur le fond de ce qui était autrefois la mer. L'absence de l'eau nous révèle le monde sous-marin et un paysage quasi surréaliste. On peut même voir une ressemblance assez frappante entre l'illustration de la couverture par Jansson et certaines œuvres de Salvador Dalí - pour exemple, dans Tentation de saint Antoine, des éléphants ont de longues jambes, presque comme les échasses avec lesquelles les enfants s'amusent à marcher. De même,

17 Michel de Montaigne, Essais III. 2, in Euvres complètes, A. Thibaudet et M. Rat (dir.), Paris, Gallimard, 1962, p. 782.

18 Tove Jansson, Kometjakten, Helsingfors, Schildts, 1946 ; Tove Jansson, Kometen kommer, Helsingfors, Schildts, 1968 
on peut se remémorer les paroles du fou dans Le gai savoir de Nietzsche quand il crie : «Où est allé Dieu ? s'écria-t-il, je veux vous le dire! Nous l'avons tué, - vous et moi! Nous tous, nous sommes ses assassins! Mais comment avons-nous fait cela ? Comment avons-nous pu vider la mer ? Qui nous a donné l'éponge pour effacer l'horizon ? Qu'avons-nous fait lorsque nous avons détaché cette terre de la chaîne de son soleil ? $"{ }^{19}$ La mer vidée est une figure de l'absence de Dieu, de l'absence de sens.

Dans Trollvinter aussi, la transformation de l'élément aquatique est surprenante pour Moumine le Troll : quand il se réveille, le monde est gelé ou sous la neige. On voit la mer comme normalement on ne la voit pas : Too-ticki montre à Moumine une cave sous la glace, presque tel un autre monde avec une autre lumière. La mer est imprévisible, prenant toujours des formes nouvelles et surprenantes. Et c'est ce qui inspire à Too-ticki cette phrase célèbre : "Tout est très incertain et c'est précisément ce qui me rassure ${ }^{20}$, qui peut être lue dans les Essais de Montaigne, ou plutôt dans un texte que Montaigne mentionne souvent, les Hypotyposes pyrrhoniennes de Sextus Empiricus, le plus important sur le scepticisme pyrrhonien dans l'Antiquité et à la Renaissance. C'est une phrase qui semble porter toute la sagesse de l'ancienne philosophie sceptique et sa recherche de l'ataraxie, la tranquillité de l'esprit. Pour Montaigne, le monde est une «branloire pérenne ", mais l'attitude qu'on doit cultiver dans ce monde en mouvement perpétuel est d'abandonner toute foi dogmatique et d'affirmer la vie. Il n'y a rien de certain dans ce monde, on ne peut pas se fier aux dogmes - et c'est exactement cela qui nous donne la tranquillité de l'âme. On peut comparer les mots de Too-ticki avec ceux de Sextus Empiricus :

Après avoir commencé à philosopher sur la distinction des représentations et sur la connaissance des vraies et des fausses, de manière à atteindre l'ataraxie, il est tombé sur une discordance d'égale force qu'il s'est abstenu, faute de pouvoir le faire, de trancher ; à cette suspension du jugement, par un heureux hasard, a fait suite l'ataraxie à l'égard de ce qui est objet d'opinion. [...] par un heureux hasard, l'ataraxie suivit pour eux la suspension du jugement, tout comme l'ombre suit le corps. ${ }^{21}$

On peut trouver la même attitude dans le livre le plus marin de la série des Moumine, Pappan och havet (Papa Moumine et la mer). Dans ce roman, papa Moumine se sent inutile et décide que toute sa famille doit déménager sur une île solitaire.

19 Friedrich Nietzsche, op. cit., $\$ 125$.

20 Tove Jansson, Un hiver dans la vallée de Moumine, trad. de K. et P. Chaplet, Paris, Nathan, 1972, p. 24.

21 Sextus Empiricus, Hypotyposes pyrrhoniennes, I, trad. de J. Grenier et G. Goron, Paris, AubierMontaigne, 1948, chap. XII, $\$ 25-29$, p. 163-164. 


\section{LE PAPA ET LA MER FÉMININE}

Pour papa Moumine, la mer est un défi hégélien, quelque chose que l'homme doit conquérir. Dans la nouvelle Hattifattarnas hemlighet (Le secret des Hatifnattes), papa Moumine suit les Hatifnattes dans leur voyage infini ${ }^{22}$. Les Hatifnattes sont pour lui des navigateurs hors pair, et il veut les rejoindre dans ce qu'il imagine être une aventure ultime. Cependant, la réalité de leur voyage quotidien est assez monotone. De plus, il se trouve incapable de communiquer avec eux et, à la fin, il quitte leur compagnie et rentre chez lui.

Comme l'a montré Maria Laakso, Pappan och havet est un livre saturé de symbolisme et de thèmes sexuels ${ }^{23}$. L'idée pour papa Moumine de conquérir l'île, avec son phare assez phallique, est clairement un projet visant à retrouver sa masculinité. Cependant, ce phare est entouré par la mer féminine, le monde incompréhensible, mais aussi fascinant. Il jette une lumière masculine sur le monde de l'obscurité féminine, profonde et humide. Papa essaie de comprendre les lois de la mer par une approche plus ou moins systématique, mais la mer lui échappe.

Moumine le Troll, d'autre part, est confronté à sa puberté dans ce livre, comme maman Moumine semble s'en rendre compte quand elle explique le comportement étrange de son fils à papa Moumine après qu'il a soudainement quitté la table pour sortir. Plus tôt, Moumine a avoué qu'il avait essayé d'attirer l'attention de deux chevaux de mer coquins, frivoles et féminins, qui se sont enfuis en riant de lui. Maman semble penser que Moumine veut simplement les revoir. Ce qu'elle ignore, cependant, c'est que Moumine a rencontré la Courabou (Mårran en suédois), l'ombre grise venant de la mer. J'ai traité ailleurs de la figure du Mårran ${ }^{24}$, et il me suffira de dire ici que les chevaux de mer et la Courabou représentent, pour Moumine le Troll, deux faces maritimes de l'autre, féminine : le côté séduisant, et celui abject et terrifiant. Moumine et papa apprennent à faire face à l'autre maritime: Moumine aide à réchauffer Courabou, et le père apprend à aimer la mer malgré son imprévisibilité.

Il faut se rappeler ici que la mer était aussi, pour papa, une source d'amour. Comme il nous le raconte dans ses mémoires, la mer lui a apporté maman Moumine qui fut jetée à terre par une vague, un peu comme Aphrodite. C'est peutêtre la raison pour laquelle papa regarde maman quand il dit : « La mer est un être

22 Tove Jansson, «Hattifattarnas hemlighet », in Det osynliga barnet och andra berättelser, Helsingfors, Schildts, 1962.

23 Maria Laakso, "Mies ja hänen saarensa. Sukupuolittuneet maisemat Tove Janssonin romaanissa Muumipappa ja meri ", in Tapion tarhoista turkistarhoille. Luonto suomalaisessa lasten- ja nuortenkirjallisuudessa, M. Laakso, T. Lahtinen et P. Heikkilä-Halttunen (éd.), Helsinki, SKS, 2011.

24 Kuisma Korhonen, «Looking through the Eyes of the Groke », Studia Culturae, 3 (25), 2015. 
immense qui est parfois de bonne humeur et d'autres fois de mauvaise humeur. Il nous est impossible de savoir pourquoi. Nous n'en voyons que la surface. Mais si on aime la mer, alors ça ne fait rien, on la prend comme elle est. $»^{25}$

On peut voir qu'à la fin du roman, papa a appris à accepter l'incertitude du monde, et - à la manière de Sextus Empiricus ou de Montaigne - la tranquillité de l'âme suit cette acceptation " comme une ombre suit le corps ». Papa a appris une philosophie écologique qui affirme que tout le monde est vivant.

\section{LA VIE DANS L'ÎLE DE KLOVHARU}

Dans les œuvres qui suivent l'épopée des Moumines, l'eau et la mer jouent souvent un rôle important. C'est le cas surtout dans Sommarboken (Le livre d'un $e^{e} t e^{26}$ et Anteckningar från en $\ddot{o}$, qui ont été inspirés par l'île de Klovharu dans le golfe de Finlande, de petite superficie, rocheuse et venteuse, sans arbres, où Tove Jansson et sa compagne Tuulikki Pietilä, dans les années 1960, ont construit une modeste hutte et passé chaque été jusqu'en 1991.

Sommarboken est un livre de fiction, mais ses personnages principaux sont reconnaissables : la vieille dame ressemble à Ham, la mère de Tove Jansson, et la petite fille Sophia à Sophia Jansson, la nièce de Tove Jansson. Entourées par la mer, la grand-mère et la fille deviennent des amies. Pendant l'été, elles contemplent la mer et tous les signes de vie qui subsistent dans les conditions difficiles de l'île. Elles apprennent aussi à connaître les pêcheurs locaux. Une nuit, elles assistent même à une opération de sauvetage : la mer est pleine de caisses de boissons illicites que les îliens tentent d'attraper depuis leurs bateaux.

Anteckningar från en ö est le dernier livre que Tove ait écrit. Illustré par Tuulikki Pietilä, il raconte l'histoire de leur vie dans l'île de Klovharu à partir des années 1960 jusqu’à leur dernier été, en 1991. Au début, il y a juste le rêve de vivre quelque part loin de tout, au milieu de la mer. Elles rencontrent ensuite un pêcheur local, Brunström, prêt à les aider. Avec d'autres locaux, elles construisent une hutte, sans autorisation officielle. Là, Tove et Tuulikki passent leurs étés, à l'écart des obligations et d'un public trop enthousiaste. Elles apprennent à entretenir leur bateau, à attraper du poisson avec des filets et d'autres techniques nécessaires pour survivre. Elles sentent que la mer est leur amie, même par temps orageux. Elles apprennent aussi à connaître les mouettes locales qui ont chacune leur propre personnalité.

25 Tove Jansson, Papa Moumine et la mer, trad. de C. Tabourin, Paris, Le Livre de poche (jeunesse), 1986, p. 221-222.

26 Tove Jansson, Sommarboken, Helsingfors, Schildts, 1972. 
Cependant, arrive finalement le moment où la vie sur l'île devient trop dure pour des personnes âgées comme Tove et Tuulikki. Leur relation à la mer change. Comme le confesse Tove, elle a commencé à craindre la mer :

Et le dernier été est arrivé quelque chose d'inexcusable ; j'ai eu peur de la mer. Les grandes vagues n'avaient plus rien à voir avec l'aventure, mais seulement avec l'angoisse et la responsabilité pour le bateau et d'ailleurs pour tous les bateaux qui sortent par mauvais temps. C'était injuste - la mer avait toujours, même dans mes cauchemars, été mon grand salut. Quand le danger guette, on plonge pour s'en aller en nageant, on se trouve en sécurité et ne revient jamais. Cette peur était comme une trahison - la mienne. ${ }^{27}$

Tove a honte de sa peur, elle se sent même coupable de trahison. La mer avait toujours été son alliée, l'aidant à se mettre à l'abri du monde. Tove et Tuulikki décident donc de quitter l'île.

Il n'est pas difficile de comprendre que Tove Jansson ne fait pas que dire au revoir à son île bien-aimée, mais aussi à sa carrière d'écrivain. La mer dans toute sa beauté et sa dangerosité représente pour elle le côté créatif de sa vie, la source d'imagination et d'aventure. Comme elle doit quitter l'île, elle doit accepter la fin de l'écriture et, irrévocablement, la fin de sa vie.

Le dernier chapitre du livre est émouvant. Ayant nettoyé la maison pour la dernière fois, Tuulikki trouve un vieux cerf-volant. Sans beaucoup d'espoir, elle l'expose au vent, et à la grande surprise des deux femmes, le vieux cerf-volant se gonfle d'air et s'envole vers la mer : " Juste pour voir, elle a donné un coup à la queue et à ce moment-là, précisément, une bourrasque est arrivée emportant le cerf-volant avec elle vers les hauteurs. Il a poursuivi sa route au loin vers le golfe de Finlande. $»^{28}$

La dernière page comporte une illustration de Tuulikki Pietilä qui montre le cerf-volant dans le ciel avec les mouettes. Libre enfin, il est comme l'âme humaine dans son ultime voyage.

\section{L'OCÉAN D'INTERPRÉTATIONS}

Pour Jansson, la mer n'est pas seulement une scène d'action. C'est une métaphore de la vie, un monde en perpétuel mouvement, un univers de non-savoir.

27 Tove Jansson, Anteckningar från en ö, op. cit., p. 90 : « Och den sista sommaren hände något oförlåtligt; jag blev rädd för havet. Stora vågor hade inte längre med äventyr att göra, bara ängslan och ansvar för båten och förresten alla båtar som rör sig ute på sjön $i$ hårt väder. Det var orättvist - till och med $i$ mardrömmarna hade havet alltid varit den osvikliga räddningen; faran är efter en men man hoppar i och simmar bort och är i säkerhet och kommer aldrig mer tillbaka. Den där rädslan kändes som ett förräderi - mitt eget. " (Notre traduction)

28 Ibid., p. 102 : «Bara för ro skull gav den lite fart i svansen och just då kom en vindil farande och tok draken med sig och den flög högt, rakt upp, och fortsatte långt ut över Finska viken. " (Notre traduction) 
Comme pour Montaigne, la réalité est toujours insaisissable pour Jansson. Rien n'est certain - même le fait que rien n'est certain.

Les interprétations que j'ai proposées ici ne sont que des suggestions, des idées sans fondement permanent. Les œuvres de Tove Jansson sont, en effet, comme la mer que papa Moumine essaie désespérément d'interpréter et de comprendre en utilisant son approche "scientifique " - jusqu'à ce qu'il apprenne à accepter qu'on peut aussi vivre avec les choses sans les interpréter, en les aimant tout simplement : "Nous n'en voyons que la surface. Mais si on aime la mer, alors ça ne fait rien."

Les limites de cet article ne me permettent pas d'aller plus loin que la surface du texte. Il y a beaucoup de choses dans les œuvres de Jansson qui nous restent cachées, enfouies dans les profondeurs. Dans l'épopée des Moumines, on peut toujours ouvrir de nouvelles possibilités interprétatives, être confronté à des visions que l'on ne voyait pas avant, mais aucune d'entre elles n'est définitive ou ne constitue une vérité allégorique bien délimitée. On peut dire des récits de Tove Jansson ce que le sage Too-ticki dit dans Trollvinter : "Tout est très incertain et c'est précisément ce qui me rassure." 